\title{
A Cross-layer Design for QoS support in Cognitive Radio Sensor Networks for Smart Grid Applications
}

\author{
Ghalib A. Shah $\uparrow$, Vehbi C. Gungorłand Ozgur B. Akan $\dagger$ \\ $\dagger$ Next-generation and Wireless Communication Laboratory (NWCL), \\ Department of Electrical and Electronics Engineering, Koc University, Istanbul, Turkey \\ $\ddagger$ Department of Computer Engineering, Bahcesehir University, Istanbul, Turkey
}

\begin{abstract}
In this paper, we propose a cross-layer design to meet the QoS requirements for smart grids employing the cognitive radio sensor networks for their control and monitoring operations. Existing routing protocols pertaining to QoS support are not able to simultaneously handle traffic of different characteristics present in smart grids. Therefore, considering the traffic heterogeneity of smart grid applications exhibiting diverse QoS requirements, a set of priority classes is defined in order to differentiate the traffic for the respective service. Specifically, the problem is formulated as a weighted network utility maximization (WNUM) whose objective is to maximize the weighted sum of flows service. A cross-layer heuristic solution is provided to solve the utility optimization problem by performing joint routing, dynamic spectrum allocation and medium access. Performance of the proposed protocol is evaluated using $n s-2$, which shows that the number of flows belonging to each class are served according to their weight fraction with their respective data rate, latency and reliability requirement.
\end{abstract}

Index Terms-Cognitive radio sensor networks (CRSN), crosslayer QoS, smart grids.

\section{INTRODUCTION}

Cognitive radios are emerging as one of the key technologies for future wireless communication due to the scarcity of the spectrum. The usage of current allocated spectrum has been witnessed as inefficient where the licensed users, also known as primary users (PUs), do not utilize their bands continuously, thus providing an opportunity to use them. Therefore, cognitive radios introduce a second class of lesser privileged users, called the secondary users (SUs), to exploit such opportunities by dynamically accessing the available spectrum without preferably causing interference to the PUs transmission. Such a dynamic access approach not only brings the potential advantage of efficient spectrum utilization but also seeks for the part of spectrum that has lesser interference due to the propagation environment.

Wireless sensor networks (WSNs) are recently gaining popularity to realize smart grids for power systems [1], [5]. Smart grids have revolutionized the power systems through the advantages of increased renewable energy usage and reduced greenhouse gas emissions, increased energy efficiency via better balance of supply and demand, and improved security and reliability via fast and effective response to energy generation and consumption fluctuations and catastrophic events [1]. The

G. A. Shah was supported in part with V.C. Gungor by the EU FP7 Marie Curie IRG under grant PIRG05-GA-2009-249206 and with O. B. Akan by TUBITAK, Turkey, Career Award under grant \#110E249. key design challenge in smart grid is to support reliable and real-time data delivery for a variety of heterogeneous sensing and actuating devices installed at consumer sites, energy meters, high-volt transformers, high-power motors, grid stations, generators, etc [4]. To address this need, WSNs employing the cognitive radio technology that forms a cognitive radio sensor networks (CRSN) [2], are sought to be the most promising solution for smart grid applications. CRSNs provide reliable data delivery with the required QoS support under the unfavourable propagation conditions of power systems. Moreover, the deployment of nodes is highly non-uniform in smart grids that does not usually expect the path redundancy unlike the traditional WSNs that exploits the redundancy of paths to overcome the nodes failure for resilient data delivery. The deployment of sensor nodes for smart grid has been largely unexplored due to its unique challenges and therefore, this paper considers the smart grid as one of its potential applications that can ultimately overcome its communication challenges in harsh noisy environment.

Very recently, smart grids have gained the interest of researchers and therefore, very few solutions exist in the literature that address the unique challenges of smart grid. A QoS routing algorithm proposed in [6], considers merely the price signalling application of smart grid that maximizes the utility function of power consumption at the consumer premises. However, it ignores the impact of external noise on transmission reliability and traffic heterogeneity present largely due to a variety of applications running in smart grid. There also exist a number of routing and MAC protocols [3], [7], [8], [9], [13] for cognitive radio ad hoc networks but they cannot be employed in smart grids mostly due to the lack of QoS support for heterogeneous traffic, which is formidable for smart grid applications. Apparently, there is an urgent need of designing a new spectrum and environment aware distributed routing protocol for CRSN.

This paper proposes a cross-layer and dynamic spectrum access framework to prevail the challenges posed in smart grid and defines a novel QoS architecture for smart grid applications. It differentiates the traffic into different QoS classes characterized in terms of data rate, latency and channel reliability. The problem is modelled as a weighted network utility maximization (WNUM) with the objective of maximizing the weighted service of traffic flows belonging to different classes. A cross-layer heuristic solution is provided that jointly 
optimizes the routing, medium access and physical layer functions. The routing protocol is an on-demand distributed algorithm that interacts with the MAC and physical layer to select a suitable channel satisfying the needs of subject flow in terms of channel interference and capacity, and prioritizing the transmission by setting the contention window size of MAC according to the class priority. In particular, it defines a routing frame period structure to control the operations of routing, MAC and physical layer. Hence the proposed solution is tailored to unique characteristics of the power system environment to meet the application-specific QoS requirements.

The remainder of the paper is organized as follows. Basic assumptions are made in Section II that also provides formal definition to the problem. The proposed cross-layer QoS routing is presented in Section III. Detailed performance evaluation and simulation results are discussed in Section IV. Finally, in Section V we draw the main conclusion.

\section{NETWORK MODEL}

In this section, we discuss the basic assumptions made in designing the cross-layer routing protocol and formulate the problem definition for the proposed work. We assume that the nodes are randomly deployed and employ single radio that switches among various traffic channels as well as a predefined common control channel. Every transmitter always transmits at a fixed transmission power level $P_{t}$. Hence, there will be a fixed interference range for a channel $h$, which is typically 2 to 3 times of the transmission range [15] for each channel. Note that the transmission and interference ranges are both channel-dependent. Let $\mathcal{H}_{u}$ be the set of channels sensed

TABLE I

PROPOSED QOS CLASSES, WHERE THE PARAMETERS ARE DEFINED OVER THE LEVELS $0=$ UNIMPORTANT, $1=$ LOW, $2=$ HIGH AND $3=$ CRITICAL.

\begin{tabular}{|l||l|l|l|}
\hline Class & $\beta$ & $\tau$ & $\alpha$ \\
\hline \hline Real-time/Reliable (XRtRe) & 1 & 3 & 3 \\
\hline Real-time/Non-reliable (XRtX) & 1 & 3 & 1 \\
\hline Non real-time/Reliable (XXRe) & 1 & 0 & 3 \\
\hline $\begin{array}{l}\text { High-rate/Real-time/Non-reliable } \\
\text { (HRaHRtX) }\end{array}$ & 2 & 2 & 1 \\
\hline $\begin{array}{l}\text { Critical-rate/Non real-time/Reliable } \\
\text { (RaXRe) }\end{array}$ & 3 & 0 & 3 \\
\hline
\end{tabular}

available by a node $n_{u}$ at any time instant $t$, slotted over $\{0,1,2, \ldots\}$, with a corresponding interference power set of $\mathcal{I}_{u}(t)=\left\{I_{1}^{u}, I_{2}^{u}, \ldots, I_{\left|\mathcal{H}_{u}\right|}^{u}\right\}$. The time varying capacity on each of these channels at node $n_{u}$ is $\mathcal{B}_{u}(t)=\left\{b_{1}^{u}, b_{2}^{u}, \ldots, b_{\left|\mathcal{H}_{u}\right|}^{u}\right\}$. The capacity of a channel $h$ of bandwidth $\delta$ is limited due to the interference in the power system and is computed as

$$
b_{h}^{u}(t)=\delta \log \left(1+K \cdot \gamma_{\overrightarrow{u v}} / I_{h}^{v}\right)
$$

where, $K$ is the limiting factor due to the bit error rate and is obtained as $K=-1.5 / \log (5 \times B E R)$, which is proved to be a good model for modulation schemes, such as, MQAM with constellation size greater than or equal to 4 [15]. The signal strength $\gamma_{\overrightarrow{u v}}$ received at node $n_{v}$ from a node $n_{u}$ transmitting at a fixed power $P_{t}$ and antenna gain $G_{\overrightarrow{u v}}$ is $P_{t} G_{\overrightarrow{u v}}$.
The data traffic in smart grids generally comprise of various types, such as, real-time pricing, supply and demand control, smart meter readings, periodic monitoring of system components, equipment fault diagnostic and control signalling of actuators, etc. In order to support QoS for the heterogeneous traffic, we classify it according to its characteristics and requirement in terms of, data rate $(\beta)$, latency $(\tau)$ and reliability $(\alpha)$. Here, data rate is presumed to be in bps, latency as end-to-end packet delay and reliability as bit error rate while transmitting it through a specific link on the selected route.

Let $\mathcal{C}$ be the set of priority classes, where each class is attributed by QoS parameters $\beta, \tau$ and $\alpha$. Each class $c \in \mathcal{C}$ is bounded to some minimum and maximum threshold values for these attributes $\left\{\left(0, \beta_{\max }[c]\right),\left(\tau_{\min }[c], \tau_{\max }[c]\right),\left(\alpha_{\min }[c], \alpha_{\max }[c]\right)\right\}$.

Similarly, there may exist an arbitrary number of flows in each class such that $F_{c}(t)$ is the set of flows in class $c$ at time slot $t$. As a result, the set of flows $\mathcal{F}$ present in the entire network is, $\mathcal{F}=\cup_{c \in \mathcal{C}} F_{c}$. Hence, the $i^{\text {th }}$ flow $f_{i}^{c}\left(\beta_{i}, \tau_{i}, \alpha_{i}\right)$ belonging to a class $c$ is privileged to use the respective service, where $0<\beta_{i} \leq \beta_{\max }[c], \tau_{\min }[c] \leq \tau_{i} \leq \tau_{\max }[c]$ and $\alpha_{\min }[c] \leq \alpha_{i} \leq \alpha_{\max }[c]$. The utility function for set of flows associated with a class $c \in \mathcal{C}$ is obtained as

$$
U_{c}\left(F_{c}\right)=\sum_{f \in F_{c}} \omega_{c} u(f)
$$

where, $u(f)$ is the utility function of flow $f$ and $\omega_{c}$ is the weighting factor of class $c$ to be privileged in data delivery and $\omega_{c} \in \Omega=\left\{\omega_{1}, \omega_{2}, \ldots, \omega_{|\mathcal{C}|}\right\}$. Every flow $f_{i}$, regardless of its class, has a source $s$ and destination $d$ pair and therefore a corresponding path $\mathcal{P}_{i}=\{\overrightarrow{s u}, \overrightarrow{u v}, \ldots, \overrightarrow{y d}\}$, where $s, u, v$, $y$ and $d$ are the members of set $\mathcal{N}$ of network nodes. The set of all paths established for the running flows for which the sources have received route reply is $\mathcal{P}$. Denote the control variable of channel selection as $x_{h}^{i}$ that takes the value 1 if the channel $h$ has the capacity to meet the data rate and reliability requirement of flow $f_{i}, 0$ otherwise. We now formulate a problem, whose objective is to maximize the utility of the network flows service globally for all $t \in\{0,1,2, \ldots\}$ subject to the flow rate, latency and reliability constraints as

\section{Maximize:}

$$
\sum_{c \in C} \sum_{i=1}^{\left|F_{c}\right|} \omega_{c} u_{i}^{c}\left(\beta_{i}, \tau_{i}, \alpha_{i}\right), \quad \omega_{c} \in \Omega
$$

\section{Subject to:}

$$
\begin{aligned}
& x_{i}^{h} \beta_{i} \leq b_{h}^{u}, \quad 0<i \leq|\mathcal{F}|, \forall h \in \mathcal{H}_{u} \cap \mathcal{H}_{v}, \overrightarrow{u v} \in \mathcal{P}_{i} \\
& \sum_{j=1}^{|\mathcal{P}|} x_{j}^{h}=0, \quad j \neq i, \quad \forall \overrightarrow{y z} \in \mathcal{P}_{j},(y, z) \in \mathcal{N}_{u} \cup \mathcal{N}_{v} \\
& x_{i}^{h} \alpha_{i} \leq\left(1-B E R\left(I_{h}^{v}\right)\right), \quad 0<i \leq|\mathcal{F}|, \\
& \forall h \in \mathcal{H}_{u} \cap \mathcal{H}_{v}, \overrightarrow{u v} \in \mathcal{P}_{i} \\
& \tau_{i} \geq \sum_{\overrightarrow{u v} \in \mathcal{P}_{i}} \overline{\tau_{\overrightarrow{u v}}}, \quad 0<i \leq|\mathcal{F}|
\end{aligned}
$$


In the above formulation, Constraint (4) ensures that the selected channel should have sufficient capacity to meet the requirement of passing flow, while (5) prohibits the use of same channel by any of the neighbors of sender and receiver at the same time $t$. Constraint (6) makes sure that the reliability of the channel in terms of bit error rate is higher than the reliability demanded by the flow. Similarly the Constraint (7) corresponds to the selection of a path that can deliver the data within the given flow delay.

\section{CROSS-LAYER QOS FrameWORK}

In this section, we present a heuristic algorithm to the problem defined in (3). The proposed solution is a crosslayer approach to achieve the desired QoS for smart grid applications. Though, a number of applications are being realized in smart grids day by day, a rich set of applications are identified in [14] along with their diverse QoS requirements. It is therefore, essential to classify the heterogeneous traffic and support the data delivery according to the priority of each traffic class. Due to resource limitation of sensor nodes, it is not possible to maintain a service queue for each different class of traffic. Therefore, we define a limited set of traffic classes along with their appropriate attributes as given in Table I and map the set of applications according to the QoS requirement as proposed in Table II. Nevertheless, the mapping is momentary and a change in mapping does not affect the functioning of the proposed solution. The proposed routing

TABLE II

SMART GRID APPLICATIONS IDENTIFIED IN [14] AND THEIR MAPPING TO THE CORRESPONDING PROPOSED QOS CLASS.

\begin{tabular}{|l|c|}
\hline Smart grid application & Service class \\
\hline \hline Smart metering & XRtRe \\
\hline Price signalling & XRtX \\
\hline SCADA & XXRe \\
\hline Operations data & RaXRe \\
\hline Inter-site rapid response & HRaHRtX \\
\hline Distribution automation & XXRe \\
\hline Distributed management \& control & XXRe \\
\hline Video & HRaHRtX \\
\hline Surveillance & HRaHRtX \\
\hline Corporate data & RaXRe \\
\hline Corporate voice & XRtRe \\
\hline
\end{tabular}

protocol is an on-demand with lower route update frequency in contrast to the ad hoc on-demand routing protocols vastly present in the literature [10], [12]. This is due to the fact that nodes are usually immobile and the node failure rate is also very low due to the possibility of recharging and continuous energy supply in smart grids. Data delivery is performed by dividing the time into routing frames. Each routing frame consists of three periods; spectrum sensing (SS) period $T_{s s}$, control $(C T R L)$ period $\left(T_{c t r l}\right)$ and data transmission (DATA period $\left(T_{t x}\right)$. The frame period is fixed to some duration $\mathcal{T}$, while the control and transmission periods are variable within the frame duration. The control period initially spans over

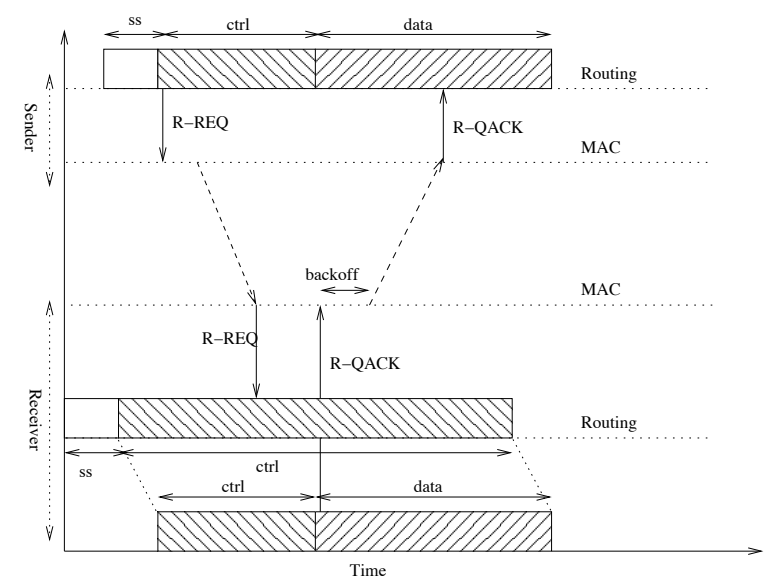

Fig. 1. Transmission of route request packet R-REQ. Receiver synchronizes its routing frame structure with the sender since its idle.

$\mathcal{T}-T_{s s}$ but skews to its minimum length $T_{c t r l}^{\min }$ as the data request is received or generated. Similarly the transmission period is initially set to 0 but is stretched to $\mathcal{T}-T_{s s}-T_{c t r l}$ for data transmission. However, if no data transmission takes place for a number of idle frames then it resets both the periods to their initial values. At the beginning of $S S$ period, routing agent switches the control to physical layer and triggers spectrum sensing for a period of $\left(T_{s s}=|\mathcal{H}| \times \Delta \times \theta\right)$ seconds, where $\Delta$ is the basic slot period and $\theta$ is the maximum number of slots to sense a channel. After SS expires, CTRL period begins in which nodes switch to a common control channel to send their periodic hello beacons, contention frames and broadcast messages.

\section{A. Route Discovery}

A node initiates route discovery whenever an event occurs or its application launches a task that composes a new flow. The node first broadcasts a route request message (R-REQ) on the control channel during the control period of its routing frame. R-REQ message contains the preferred data channel of the sender from its free channels set, which is decided according to the constraint defined by a class whose flow is under consideration. That is, a node $n_{u}$ selects a data channel $h \in \mathcal{H}_{u}$ that meets the data rate and reliability constraint of the flow for which the request is being sent. Thereafter, it switches to the channel $h$ and waits for the request acknowledgement message (R-QACK). If R-QACK message is not received within the Ack timeout period $\left(T O_{q a c k}\right)$ then it switches back to the control channel and repeats the procedure. The value of $T O_{\text {qack }}$ is set to $\Delta \times C W_{\max }[c]+\overline{R T T}$ for a flow belonging to the traffic class $c$. $C W_{\max }[c]$ represents the maximum contention window size defined for a class $c$ at MAC layer and $\overline{R T T}$ the mean round trip time measured by $n_{u}$. If R-QACK message arrives at $n_{u}$ then it sticks with its current routing frame structure until R-REP is received from the destination. Nodes which receive the R-REQ from $n_{u}$, switch to channel $h$ if found in their free channels list. Moreover, they also synchronize their routing frame structure with the sender routing frame if they are not participating 
in any transmission before and their entire frame period is used for control signalling. This is illustrated in Fig. 1, in which the receiver control period spanned over whole frame duration but synchronizes it with the sender on reception of R-REQ message. At the beginning of their DATA period, one of the recipient sends R-QACK message that also contains the average interference perceived on channel $h$ by the replying node. Note that the request may be acknowledged by more than one nodes. However, if a node intending to acknowledge the R-REQ, overhears the R-QACK for the same source by one of its neighbors, it retracts its acknowledgement message but continues to propagate the R-REQ. Hence, R-REQ continues to propagate in the network through different routes until it reaches at the destination.

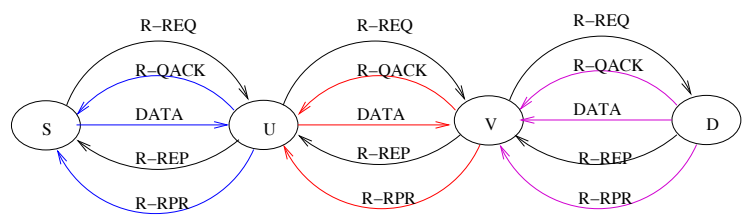

Fig. 2. Route discovery from node $s$ to $d$, where a different color denotes a different channel used for transmission. R-REQ and R-RPR uses the common control channel, while R-QACK and R-REP uses the data channel that may vary hop by hop.

Destination node responds to the first received message by sending a unicast R-REP message on the data channel recommended in the R-REQ message of previous hop node. This path is assumed to be the shortest path in terms of latency. It follows the same path of R-REQ back to the source node using the same channels. Note that the traffic channel at each hop might not be unique and may vary hop to hop as shown in Fig 2.

\section{B. MAC frame transmission}

We assume that the underlying MAC is a CSMA/CA based protocol integrated with the dynamic spectrum access (DSA) functionality to perform spectrum sensing for determining the list of available channels. When a node $n_{u}$ has data frame for transmission, after the $S S$ period is elapsed, it sets its $C T R L$ period to $T_{c t r l}^{\min }$. It also initializes its contention window $(C W)$ to $C W_{\min }[c]$ for a class $c$ data transmission and selects a random backoff value between 0 and $C W$ and runs its backoff timer. As the backoff timer expires, if it senses the medium idle then transmits channel contention request frame C-RTS containing the preferred data channel $h$ and start of $C T R L$ period, otherwise it increases its $C W$ and runs backoff. It continues until C-RTS is successfully sent.

When a node $n_{v}$ receives the channel contention frame C-RTS for a data request on its control channel, it replies to $n_{u}$ with channel contention response frame C-CTS and synchronizes its routing frame with the $n_{u}$ if its DATA period is not enabled yet. Node $n_{v}$ switches to the data channel $h$ recommended by $n_{u}$ in its C-RTS frame and waits for a data packet during the $D A T A$ period. If it receives a data frame, it sends data acknowledgement ACK frame to $n_{u}$ and remains synchronized with $n_{u}$ in the subsequent routing frame.
However if $n_{v}$ does not receive a data message during the DATA period, it is free to synchronize with any other node that sends it to RTS frame. Note that if a node $n_{v}$ has its own data frame of higher or same priority class for transmission then it initiates its own frame structure for channel contention.

\section{Class priority}

In order to prioritize different classes according to the assigned weight, different values of minimum contention window size are determined. This allows to use backoff period according to the priority of class where a lower priority gets a larger window size and in turn longer backoff period. Hence, the collaborative MAC/Routing scheme exhibits the support of different priority classes. The routing protocol makes sure that a suitable channel having sufficient capacity and constrained bit error rate is selected, while MAC provides priority in transmission in the form of different backoff intervals. Thus for a class $c \in \mathcal{C}$ and the minimum contention window limit of $C W_{\min }$, we obtain a minimum window size for $c$ as

$$
C W_{\min }[c]=C W_{\min }\left(1+W\left(1-\frac{\omega_{c}}{\sum_{\omega \in \Omega} \omega}\right)\right)
$$

where, $W$ is the scaling constant and setting it to larger value provides more strict priorities to classes and vice versa. Moreover, the base station also sends feedback to the source node if the data received does not acquire the service pertinent to its QoS class. As a result, source node updates its routing path by initiating R-REQ again.

\section{PERformance Evaluation}

In this section, we present the performance results of the proposed framework simulated using $n s-2$ [16]. In our experiments, we defined first four priority classes of the proposed framework with priority set $\{4,3,2,1\}$. Three are total of 12 flows, 3 per each class, where the flows in first two classes (XRtRe and XRtX) generate data at $20 \mathrm{kbps}$, in third class (XXRe) at $30 \mathrm{kbps}$ and in fourth class (HRaHRtX) at $40 \mathrm{kbps}$. Similarly three pairs of PUs generate data at $20 \mathrm{kbps}$ per pair on the fixed channels 2, 3 and 4, while SUs dynamically access the available channels from 2 to 10 . Channel 1 is fixed as a common control channel for the SUs. The performance metric are average delay, throughput and reliability in each class.

Fig. 3 shows the average delay measured in four different classes. It can be seen that, the delay is lower in realtime classes according to their priority and it decreases as the number of potential channels are increased. However, it decreases until the number of channels are 7 and starts increasing slightly after this. This is due to the fact that benefit of higher number of channels is achieved only when the PU traffic is present on large number of channels or the channels are saturated or suffering high noise otherwise it also becomes overhead in terms of sensing and switching between large number of channels. Hence, the delay is minimum at 7 channels. Moreover, class HRaHRtX is real-time with high data rate requirement and it gets high data rate with lower delay than the non-real-time class XXRe. 


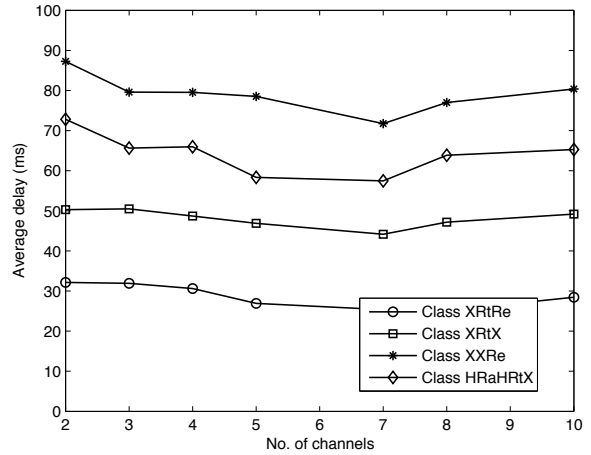

Fig. 3. Average packet delay in different classes.

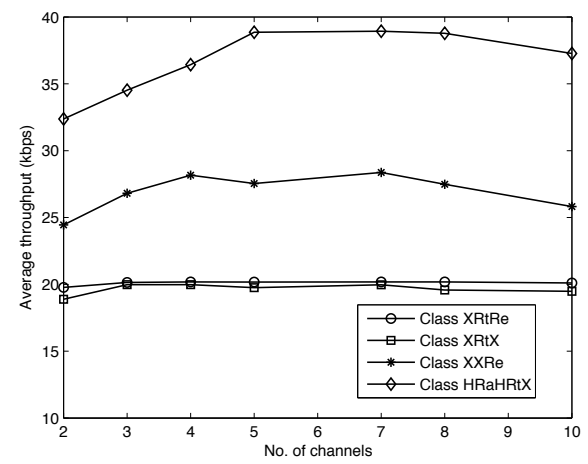

Fig. 4. Average packet throughput in different classes.

The average throughput is plotted in Fig. 4 for the same channels. Although, first two classes have the same data rate but the second class has lesser reliability requirement that reduces its data rate and thus it achieves slightly lower rate than the first class XRtRe. This is also justifies in Fig. 5 that presents the reliability of the classes. Here, the average packet delivery ratio of XRtX is about $13 \%$ lower than XRtRe that reduces its throughput. The class XXRe is the reliable along with class XRtRe that has higher reliability of about $95 \%$, slightly lesser than XRtRa due to its lower priority metric. Hence, the highest priority class XRtRe has the least delay with the highest reliability. The class HRaHRtX achieves highest data rate with lower delay than the non real-time class XXRe, which shows that the framework provides QoS to each flow according to its specified class.

\section{CONCLUSIONS}

In this paper, we propose a framework that prevails the challenges of hostile propagation conditions and traffic heterogeneity in smart grids by employing cognitive radio sensor networks with QoS support. The framework defines a novel QoS architecture for smart grid applications that differentiates the traffic into different QoS classes characterized in terms of data rate, latency and channel reliability. The problem is modelled as a weighted network utility maximization (WNUM) with the objective of maximizing the weighted service of traffic flows belonging to different classes. A cross-layer heuristic

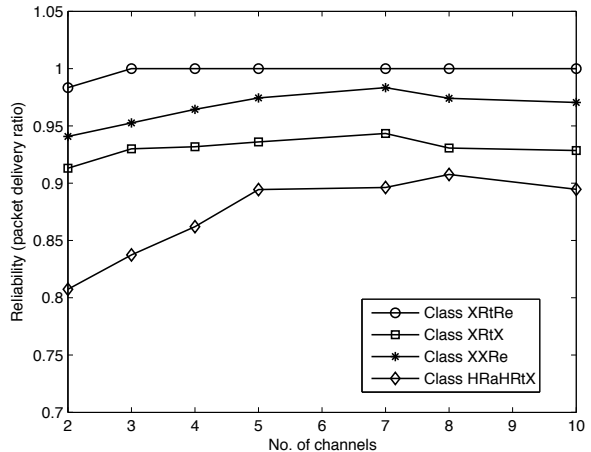

Fig. 5. Average packet reliability in different classes.

solution is provided that jointly optimizes the routing, medium access and physical layer functions. and latency. Performance of the proposed protocol is evaluated using $n s-2$, which shows that the number of flows belonging to each class are served according to their weight fraction with their respective data rate, latency and reliability requirement.

\section{REFERENCES}

[1] V. C. Gungor, B. Lu and G. P. Hancke, "Opportunities and Challenges of Wireless Sensor Networks in Smart Grid," IEEE Tran. on Industrial Electronics, vol. 57, no. 10, pp. 3557-3564, 2010.

[2] O. B. Akan, O. B. Karli, and O. Ergul, "Cognitive Radio Sensor Networks," IEEE Network, vol. 23, no.4, pp. 34-40, Jul 2009.

[3] A.O. Bicen, V.C. Gungor, O.B. Akan, "Delay-Sensitive and Multimedia Communication in Cognitive Radio Sensor Networks," Ad Hoc Networks Journal, 2011.

[4] V.C. Gungor, et al. "Smart Grid Technologies: Communication Technologies and Standards," in IEEE Transactions on Industrial Informatics, vol 7, no. 4, pp. 529-539, 2011.

[5] V.C. Gungor, "Multimedia Wireless Sensor Networks for Smart Grid Applications," in IEEE COMSOC MMTC E-Letter, vol. 6, no. 12, pp. 9-11, 2011.

[6] H. Li and W. Zhang, "QoS Routing in Smart Grid," in Proc. of IEEE Globecom, 2010

[7] L. Ding et. al., "Cross-Layer Routing and Dynamic Spectrum Allocation in Cognitive Radio Ad Hoc Networks," IEEE TVT vol. 59, no. 4, pp. 1969-1979, 2010.

[8] H. Su and X Zhang, "Cross-Layer Based Opportunistic MAC Protocols for QoS Provisioning Over Cognitive Radio Wireless Networks," IEEE JSAC vol. 26, no. 1, pp. 118-129, 2008.

[9] R. Hincapie, J. Tang, G. Xue and R. Bustamante, "QoS Routing in Wireless Mesh Networks with Cognitive Radios," IEEE GLOBECOM'08. pp. 1-5, Nov 2008.

[10] A. Abbagnale, F. Cuomo, "Gymkhana: A Connectivity-Based Routing Scheme for Cognitive Radio Ad Hoc Networks," IEEE INFOCOM'10, pp.1-5, Mar 2010.

[11] A. Al-Fuqaha et. al. "Opportunistic Channel Selection Strategy for Better QoS in Cooperative Networks with Cognitive Radio Capabilities,' IEEE JSAC, vol.26, no.1, pp.156-167, Jan. 2008.

[12] K. R. Chowdhury, and M.D. Felice, "Search: A routing protocol for mobile cognitive radio ad-hoc networks," Computer Communications vol. 32, no. 18, pp. 1983-1997, 2009.

[13] A. O. Bicen, O. B. Akan, "Reliability and Congestion Control in Cognitive Radio Sensor Networks," Ad Hoc Networks Journal, vol. 9, no. 7, pp. 1154-1164, 2011.

[14] Y.-H. Jeon, "QoS Requirements for the Smart Grid Communications System," International Journal of Computer Science and Network Security, vol. 11 no. 3, pp. 89-94, 2011.

[15] G. J. Foschini and J. Salz, "Digital communications over fading radio channels," Bell Syst. Tech. J., vol. 62, pp. 429-456, Feb. 1983.

[16] UC Berkeley, LBL, USC/ISI and Xerox PARC, "The Network Simulator ns-2," The VINT Project, http://www.isi.edu/nsnam/ns/. 